\title{
Entanglement negativity via replica trick: a Quantum Monte Carlo approach
}

\author{
Chia-Min Chung, ${ }^{1}$ Vincenzo Alba, ${ }^{2}$ Lars Bonnes, ${ }^{3}$ Pochung Chen,,${ }^{1,4}$ and Andreas M. Läuchli ${ }^{3}$ \\ ${ }^{1}$ Department of Physics and Frontier Research Center on Fundamental and Applied Sciences of Matters, \\ National Tsing Hua University, Hsinchu 30013, Taiwan \\ ${ }^{2}$ Department of Physics and Arnold Sommerfeld Center for Theoretical Physics, \\ Ludwig-Maximilians-Universität München, D-80333 München, Germany \\ ${ }^{3}$ Institute for Theoretical Physics, University of Innsbruck, A-6020 Innsbruck, Austria \\ ${ }^{4}$ Physics Division, National Center for Theoretical Sciences, Hsinchu 30013, Taiwan
}

(Dated: October 31, 2018)

\begin{abstract}
Motivated by recent developments in conformal field theory (CFT), we devise a Quantum Monte Carlo (QMC) method to calculate the moments of the partially transposed reduced density matrix at finite temperature. These are used to construct scale invariant combinations that are related to the negativity, a true measure of entanglement for two intervals embedded in a chain. These quantities can serve as witnesses of criticality. In particular, we study several scale invariant combinations of the moments for the $1 \mathrm{D}$ hard-core boson model. For two adjacent intervals unusual finite size corrections are present, showing parity effects that oscillate with a filling dependent period. These are more pronounced in the presence of boundaries. For large chains we find perfect agreement with CFT. Oppositely, for disjoint intervals corrections are more severe and CFT is recovered only asymptotically. Furthermore, we provide evidence that their exponent is the same as that governing the corrections of the mutual information. Additionally we study the 1D Bose-Hubbard model in the superfluid phase. Remarkably, the finite-size effects are smaller and QMC data are already in impressive agreement with CFT at moderate large sizes.
\end{abstract}

Introduction. - The quest for universality has long been a driving research theme at the border between condensed matter and quantum field theory. Recently, much progress has been achieved due to the deep relation between conformal field theory (CFT) and quantum entanglement [1-4].

Given a bipartition of a system (in a pure state $|\psi\rangle$ ) into two parts $A$ and $B$, a measure of their mutual entanglement is the von Neumann entropy $S_{1} \equiv \operatorname{tr} \rho_{A} \log \rho_{A}$, with $\rho_{A} \equiv$ $\operatorname{tr}_{B}|\psi\rangle\langle\psi|$ the reduced density matrix for $A$. Alternatively, the so-called Renyi entropy $S_{n} \equiv-1 /(n-1) \log \operatorname{tr} \rho_{A}^{n}[5-7]$ are also valid entanglement measures.

It is now well established that the entropies contain universal information about 1D critical systems, namely the central charge [2-4, 8] of the underlying CFT. Moreover, if subsystem $A$ consists of two (or many) disjoint intervals, as $A \equiv A_{1} \cup A_{2}$, the mutual information, $I_{A_{1}: A_{2}} \equiv S_{A_{1}}+S_{A_{2}}-S_{A_{1} \cup A_{2}}$, depends on the full operator content of a CFT [7, 9-20].

However, as the subsystem $A_{1} \cup A_{2}$ is generally in a mixed state, the mutual information is not a measure of their entanglement but of all (quantum and classical) correlations between $A_{1}$ and $A_{2}$ [21]. Their entanglement, instead, can be quantified via the logarithmic negativity $\mathcal{E}$ [22]

$$
\mathcal{E} \equiv \log \left\|\rho_{A}^{T_{2}}\right\|=\log \operatorname{tr}\left|\rho_{A}^{T_{2}}\right| .
$$

Here $\rho_{A}^{T_{2}}$ is the partially transposed reduced density matrix with respect to $A_{2}$ (formally $\left\langle\varphi_{1} \varphi_{2}\left|\rho_{A}^{T_{2}}\right| \varphi_{1}^{\prime} \varphi_{2}^{\prime}\right\rangle \equiv$ $\left\langle\varphi_{1} \varphi_{2}^{\prime}\left|\rho_{A}\right| \varphi_{1}^{\prime} \varphi_{2}\right\rangle$, with $\left\{\varphi_{1}\right\},\left\{\varphi_{2}\right\}$ being a basis for $\left.A_{1}, A_{2}\right)$.

Unlike the entropy, which contains non universal contributions, the negativity $\mathcal{E}$ is fully universal at a quantum critical point and, therefore, a useful tool to distinguish between different universality classes. This was originally argued on the basis of DMRG calculations [23, 24], and it has been shown analytically only recently using CFT techniques [25, 26]. Furthermore, the negativity is attracting increasing attention in $D>1$ as an alternative indicator of topological order [27, 28].
In this work we investigate the scaling behavior of $\operatorname{tr}\left(\rho_{A}^{T_{2}}\right)^{n}$, i.e. the $n$-th moment of $\rho_{A}^{T_{2}}$, from which the negativity can in principle be obtained as the analytic continuation $[25,26] \mathcal{E}=\lim _{n \rightarrow 1} \operatorname{tr}\left(\rho_{A}^{T_{2}}\right)^{n}$ ( $n \in \mathbb{N}$ even $)$. Although not being proper entanglement measures, in $1 \mathrm{D}$ they provide universal information about critical systems. Specifically, for two adjacent intervals (cf. Fig. 1 (a)) their scaling behavior depends solely on the central charge, whereas for disjoint ones (Fig. 1 (c)) it can potentially reveal complete information about a CFT $[25,26]$.

Summary of results. - We provide a novel Quantum Monte Carlo (QMC) scheme to calculate the moments of the transposed density matrix, $\operatorname{tr}\left(\rho_{A}^{T_{2}}\right)^{n}$, at finite temperature, using the replica trick (similarly to Monte Carlo approaches for the Renyi entropies [9, 17, 18, 29-39]). Our scheme generalizes that proposed in Ref. 30 using classical Monte Carlo. While only universal features can be accessed easily via classical simulations, in QMC both universal and non universal aspects can be accessed directly. For instance, temperature is a tunable parameter in QMC, whereas this is not possible, in an easy manner, within the classical Monte Carlo scheme. Thus, the QMC approach is ideal for benchmarking future finite temperature CFT results. Interestingly, it should also be possible (in principle) to reconstruct the spectrum of $\rho_{A}^{T_{2}}$ (and hence the negativity), as we have demonstrated recently for the reduced density matrix in Ref. 39 (see also Ref. 40).

Instead of considering $\operatorname{tr}\left(\rho_{A}^{T_{2}}\right)^{n}$, we introduce the combinations $r_{n}$ and $R_{n}$ (respectively for adjacent and disjoint intervals, see below for their definitions). These are scale invariant at a critical point and can be used as witnesses of exotic (topological) critical behaviors (similarly to Binder cumulants [41] for standard criticality).

To be specific, here we consider 1D hard-core bosons (at half and quarter filling) and the Bose-Hubbard chain in the superfluid phase. Both are special instances of the Luttinger liq- 

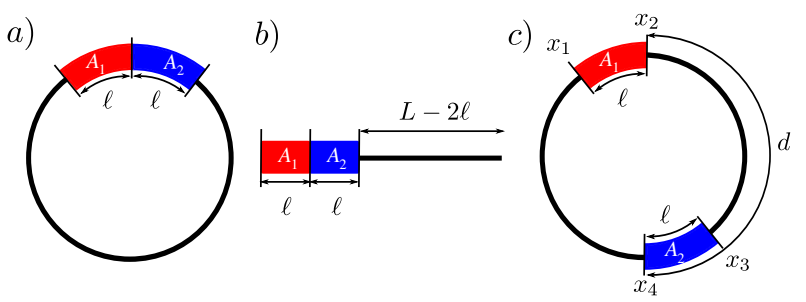

FIG. 1. Geometrical setup (chain partitions) used in this work: two adjacent intervals $\left(A_{1}, A_{2}\right)$ of equal length $\ell$ embedded in a chain (of length $L$ ) with periodic (a) and open (b) boundary conditions. (c) Two disjoint intervals.

uid, which is a $c=1$ CFT. Surprisingly, for hard-core bosons, despite integrability, it is a formidable challenge to calculate analytically $\rho_{A}^{T_{2}}$ (in contrast with the case of free bosons [42]).

At low enough temperature, we find that $r_{n}$ is in excellent agreement with CFT $[25,26]$ for large enough chains, while at small sizes unusual (in the sense of Ref. [43]) corrections are present. These arise from the local breaking of conformal invariance near the endpoints of the intervals, and are generic for entanglement-related quantities and show parity oscillations alike standard Renyi entropies [44-46]. Detailed knowledge of these corrections is imperative with respect to the application of entanglement related tools as indicators of critical behavior.

For hard-care bosons we provide convincing evidence that the leading exponent of these corrections in the two-interval case - where unusual contributions are generally stronger is $\omega_{n}^{\prime}=2 / n$. This suggests that $\omega_{n}^{\prime}=2 K_{L} / n$ in a generic Luttinger liquid (here $K_{L}$ is the Luttinger parameter) wich is the same as for the mutual information [17, 18]. Finally, in the Bose-Hubbard chain scaling corrections are smaller and we find perfect agreement with CFT predictions already at finite but large enough lattices.

Models \& observables. - We mainly consider the (integrable) 1D hard-core boson (at most one particle per lattice site) model with $L$ sites, which is defined by the Hamiltonian $\mathcal{H}=-t \sum_{i}\left(b_{i}^{\dagger} b_{i+1}+\right.$ h.c. $)$. Here $b_{i}$ are bosonic annihilation operators and $t=1$ is the hopping amplitude. In particular we work at half and quarter fillings. We also consider the 1D Bose-Hubbard model given by the Hamiltonian $\mathcal{H}=-t \sum_{i=1}\left(b_{i}^{\dagger} b_{i+1}+\right.$ h.c. $)+U / 2 \sum_{i} n_{i}\left(n_{i}-1\right)$, where $U$ is the interaction strength. Specifically we restrict ourselves to the superfluid phase at unit filling and fix $U=2$ (the Mottsuperfuid transition being at $U \approx 3.38$ [47]). The low energy properties of both models are captured by a gapless Luttinger liquid. For hard-core boson model the Luttinger parameters $K_{L}=1$, while for Bose-Hubbard model $K_{L} \approx 3.125$ at $U=2[48,49]$. For two adjacent intervals [cf. Fig. 1(a),(b)] we define (following [25]) the ratios $r_{n}(n \in \mathbb{N})$

$$
r_{n}(z) \equiv \log \left[\frac{\operatorname{tr}\left(\rho_{A_{1} \cup A_{2}}^{T_{2}=\ell}\right)^{n}}{\operatorname{tr}\left(\rho_{A_{1} \cup A_{2}}^{T_{2}=L / 4}\right)^{n}}\right],
$$

with $z \equiv \ell / L[50]$. Here the notation $\rho^{T_{2}=\ell}$ means that the partial transposition is done with respect to the degrees of

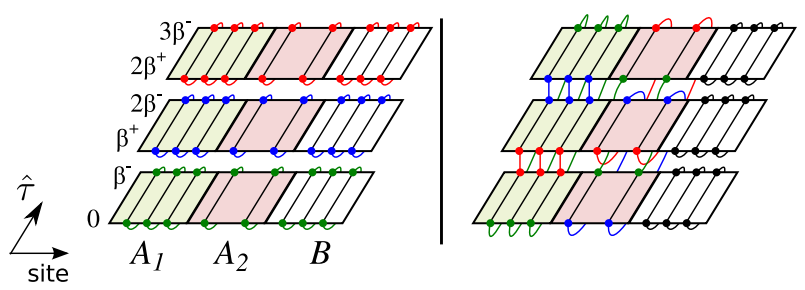

FIG. 2. Replica trick scheme for calculating the $n$th moment (here $n=3$ ) of $\rho_{A}^{T_{2}}$ in Quantum Monte Carlo (QMC) simulations. Left: three disconnected replicas (of area $L \times \beta$ ). On each replica periodic boundary conditions are used along the imaginary time direction $\hat{\tau}$. Right: Topology of $\mathcal{K}_{3}$ for two adjacent intervals. Colored links now connect points on different replicas. Regions corresponding to different intervals $A_{1}, A_{2}$ (see Fig. 1) are shaded with different colors.

freedom of subsystem $A_{2}$ of length $\ell$ (see Fig. 1 (a)(b)). For two disjoint intervals it is convenient to define $R_{n}$ as

$$
R_{n}(y) \equiv \frac{\operatorname{tr}\left(\rho_{A_{1} \cup A_{2}}^{T_{2}}\right)^{n}}{\operatorname{tr} \rho_{A_{1} \cup A_{2}}^{n}}
$$

where $y$ is the four point ratio $y \equiv\left|\left(x_{2}-x_{1}\right)\left(x_{4}-x_{3}\right)\right| / \mid\left(x_{3}-\right.$ $\left.x_{1}\right)\left(x_{4}-x_{2}\right) \mid$ [see Fig. 1(c)], and one has $\left|x_{i}-x_{j}\right| \rightarrow$ $L / \pi \sin \left(\pi\left|x_{i}-x_{j}\right| / L\right)$ (chord length) for finite chains. By construction, all the length scales and non universal factors cancel in Eqs. (2) and (3). As a consequence $r_{n}(z)$ and $R_{n}(y)$ are scale invariant quantities (for any $n, z, y$ ) at criticality apart from scaling corrections at finite $L, \ell$. Moreover, while $r_{n}(z)$ depends only on the central charge, much more universal information is contained in $R_{n}(y)$.

The moments of $\rho_{A}^{T_{2}}$ : QMC algorithm.- The moments of the partially transposed reduced density matrix $\operatorname{tr}\left(\rho_{A}^{T_{2}}\right)^{n}$ can be measured in Quantum Monte Carlo (QMC) simulations by exploiting a suitable replica representation. Given a generic lattice model, one has [25]

$$
\operatorname{tr}\left(\rho_{A_{1} \cup A_{2}}^{T_{2}}\right)^{n}=\frac{Z_{n}^{T_{2}}\left(A_{1} \cup A_{2}\right)}{Z^{n}},
$$

where $Z=\operatorname{tr} \exp (-\beta H)$ is the partition function at temperature $T=1 / \beta$, while $Z_{n}^{T_{2}}$ is defined over an ad hoc surface $\mathcal{K}_{n}$, obtained by "gluing" $n$ disconnected replicas. For $n=3$ and two adjacent intervals $\mathcal{K}_{n}$ is illustrated in Fig. 2 (right), and is formally obtained by introducing an equal-time branch cut (lying along subsystem $A$ ) at $\tau=k \beta, k=1,2, \ldots, n$ on each replica. Links crossing the branch cuts (colored links in Fig. 2 (right)) connect sites on different replicas. The "gluing" scheme is different for the two intervals $A_{1}, A_{2}$, reflecting the partial transposition on $A_{2}$.

The ratio in Eq. (4) can be sampled using a world-line based QMC. Here we use a continuous time world algorithm [5153] (extensions to other QMC schemes are straightforward), supplementing the standard world line update with a non-local move. Given that the system is on $\mathcal{K}_{n}$ [cf. Fig. 2 (right)], the move tries to cut all the world lines at $k \beta^{+}$and $k \beta^{-}$, creating new ones connecting sites at $k \beta^{+}$and $(k+1) \beta^{-}$, as in Fig. 2 (left) (note the periodicity in imaginary time). If the move is 


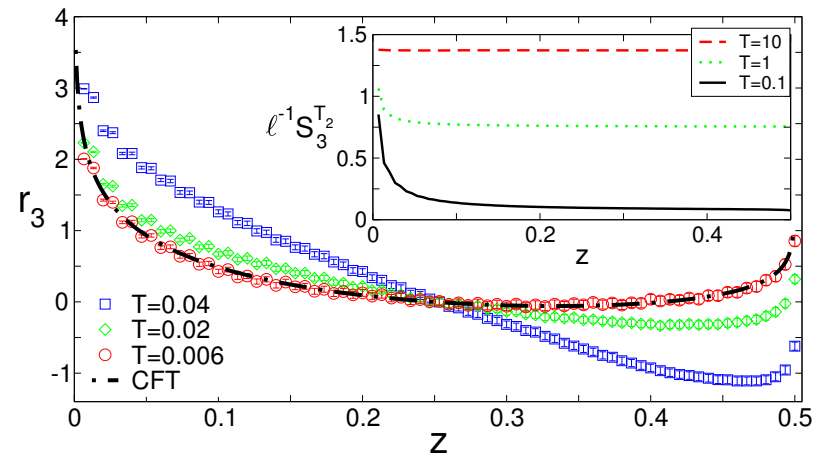

FIG. 3. Two adjacent intervals: We show QMC data for $r_{3}$ versus $z \equiv \ell / L$ for a periodic chain of length $L=150$ of hard-core bosons at half filling for different temperatures. The dashed-dotted line is the zero temperature CFT result. Note that the crossing at $z=1 / 4$ is merely due to the definition of $r_{n}$. Inset: $\ell^{-1} S_{3}^{T_{2}}$ versus $z$ (QMC data) showing that the transposed Renyi entropy exhibits a volume law already at $T \sim 1$.

possible, the global topology is changed from $\mathcal{K}_{n}$ to $n$ disconnected sheets. The inverse move from $n$ disconnected sheets to $\mathcal{K}_{n}$ is performed in a similar fashion. Finally, one measures $\operatorname{tr}\left(\rho_{A}^{T_{2}}\right)^{n}=\left\langle N_{A}^{c} / N_{A}^{\text {dis }}\right\rangle$, where $N_{A}^{c}$ and $N_{A}^{\text {dis }}$ are the total number of QMC steps happening on the connected replicas $\mathcal{K}_{n}$ and disconnected sheets respectively, and $\langle\cdot\rangle$ is the Monte Carlo average.

Upon increasing the length of $A$ as well as the replica index $n$ the transition probabilities in the global update become small, severely limiting the performance of the algorithm. To circumvent these issues we use the so called increment tricks [31, 39].

Two adjacent intervals. - As a benchmark of the algorithm we first focus on two adjacent (equal-size) intervals [cf. Fig. $1(\mathrm{a}, \mathrm{b})$ ], discussing the scaling invariant ratios $r_{n}$ $(n=3,4)$. Fig. 3 plots $r_{3}$ as function of $z \equiv \ell / L$ (data for a periodic hard-core boson chain of length $L=150$ and several temperatures). At $T=0, r_{n}(z)$ (for any $n$ ) can be obtained analytically using Eq. (2) and in any CFT one has [25, 26, 54]

$$
\begin{aligned}
& \operatorname{tr}\left(\rho_{A}^{T_{2}}\right)^{n_{e}} \propto\left(\ell_{1} \ell_{2}\right)^{-\frac{c}{6}\left(\frac{n_{e}}{2}-\frac{2}{n_{e}}\right)}\left(\ell_{1}+\ell_{2}\right)^{-\frac{c}{6}\left(\frac{n_{e}}{2}+\frac{1}{n_{e}}\right)} \\
& \operatorname{tr}\left(\rho_{A}^{T_{2}}\right)^{n_{o}} \propto\left(\ell_{1} \ell_{2}\left(\ell_{1}+\ell_{2}\right)\right)^{-\frac{c}{12}\left(n_{o}-\frac{1}{n_{o}}\right)},
\end{aligned}
$$

with $\ell_{i}$ the two intervals lengths, $n_{e}\left(n_{o}\right)$ an even(odd) integer, and $c$ the central charge. The resulting theoretical curve (after replacing $\ell_{i} \rightarrow L / \pi \sin \left(\pi \ell_{i} / L\right)$ in Eq. (5)) is plotted in Fig. 3 as a dashed-dotted line. At $T=0.006$, QMC data perfectly agree with CFT (i.e. scaling corrections are small). Interestingly, $r_{3}$ provides an effective way of extracting $c$. Indeed, fitting QMC data to Eq. (5), one obtains $c=0.98(5)$, fully compatible with $c=1$. On the other hand, finite temperature effects are already visible at $T=0.02$.

It is also instructive to consider the "transposed entropy" $S_{n}^{T_{2}} \equiv-\log \operatorname{tr}\left(\rho_{A}^{T_{2}}\right)^{n}$ (see the inset in Fig. 3). At $T \rightarrow \infty$ one expects (for the infinite chain) a thermodynamic volume law $S_{n}=S_{n}^{T_{2}}=2 \ell \log 2, \forall n$. This is already visible at $T \sim 10$ reflecting that $\rho_{A}$ is almost diagonal. Since at high tempera-

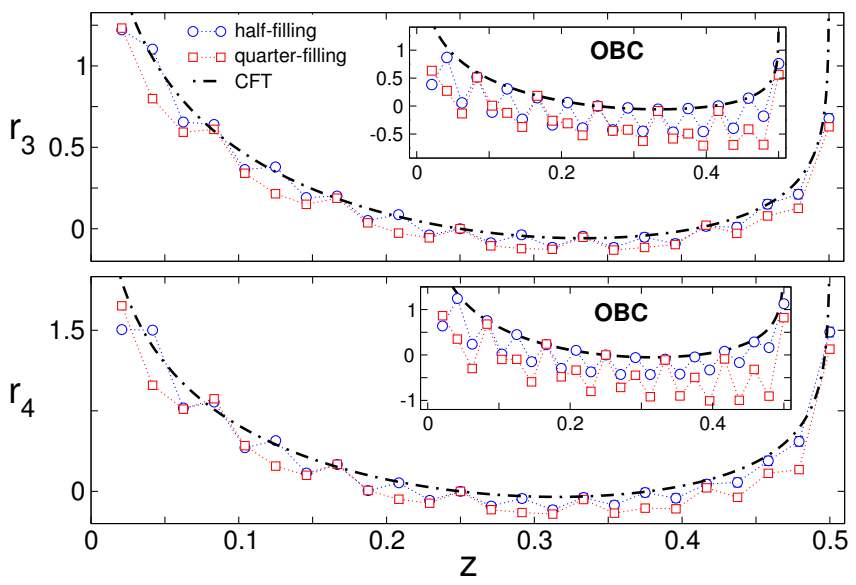

FIG. 4. Scaling corrections of $r_{3}$ and $r_{4}$ : parity and boundary effects. $r_{3}$ (top) and $r_{4}$ (bottom) from QMC as function of $z \equiv \ell / L$ at fixed $L=48$ and $T=0.01$ for a periodic chain of hard-core bosons at half (circles) and quarter (squares) filling. The dashed-dotted line is the zero temperature CFT result (same as in Fig. 3). Inset: same as in the main figure but for open boundary conditions. The amplitude of the corrections is enhanced.

ture only classical correlations survive, this implies that $S_{n}^{T_{2}}$ is not a good entanglement measure.

Unusual scaling corrections.- One intriguing feature of entanglement related quantities is that they exhibit unusual finite size scaling corrections [43]. These arise from conical singularities near the endpoints of the subsystems, and can depend on both irrelevant and relevant operators (in the renormalization group sense) of the theory, whereas usual corrections are due only to irrelevant ones.

For Luttinger liquid unusual corrections lead to parity oscillations of the Renyi entropies that can be given as [45, 46]

$$
S_{n}(\ell)-S_{n}^{C F T}(\ell)=f_{n} \cos \left(2 k_{F} \ell\right) \ell^{-\omega_{n}},
$$

with $f_{n}$ a nonuniversal amplitude and $k_{F}$ the Fermi momentum. Notably, $\omega_{n}$ depends on the Luttinger parameter $K_{L}$ as well as on the Renyi index (and thus on the global geometry) as $\omega_{n}=(2) K_{L} / n$ for the open (periodic) case.

It is natural to expect similar corrections for $S_{n}^{T_{2}}$ (and for $r_{n}(z)$ thereof). This is supported in Fig. 4 plotting QMC data for $r_{3}$ and $r_{4}$ as function of $z$ showing data for both periodic and open boundary conditions at fixed $L=48$, and half and quarter filling $\left(k_{F}=\pi / 2\right.$ and $\left.k_{F}=\pi / 4\right)$. Clearly, scaling corrections oscillate consistently with $\sim \cos 2 k_{F} \ell$ (for both open and periodic boundary conditions), in agreement with a generalization of Eq. (6) to the case of two intervals. Interestingly, as for the standard entropies [55, 56], the corrections amplitude is enhanced (for $r_{3}$ by a factor $\sim 10$ ) with open boundary conditions (cf. insets in Fig. 4).

Two disjoint intervals. - We now turn to the more complicated situation of two disjoint intervals in a periodic chain (see Fig. 1 (c)), focusing on the ratio $R_{3}(y)$ (see Eq. (3)). In the asymptotic limit (after sending all the length scales to in- 

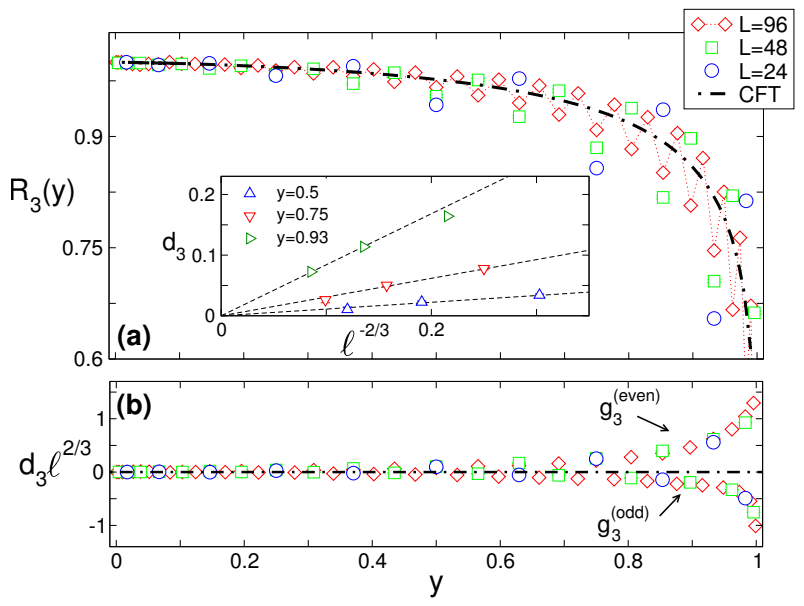

FIG. 5. 1D hard-core bosons: the ratio $R_{3}(y)$. QMC data at halffilling, chain lengths $L=24,48,96$ (periodic boundary conditions), and temperatures $T=0.24 / L$. (a) $R_{3}(y)$ vs cross ratio $y$. The dotted line highlights the oscillating behavior. The dashed-dotted line is the zero temperature CFT result. Inset: $d_{3} \equiv R_{3}^{C F T}-R_{3}$ (at fixed $y$ ) versus $\ell^{-2 / 3}, \ell$ being the intervals size. Dashed lines are one parameter fits to $\sim \ell^{-2 / 3}$. (b) Amplitude $d_{3} \ell^{2 / 3}$ of the corrections plotted versus $y$. Note the data collapse with the functions $g_{3}^{(q)}(y), q$ being the parity of $\ell$.

finity), for any model described by a CFT it is given as $[25,26]$

$$
R_{n}^{C F T}(y)=(1-y)^{\frac{c}{3}\left(n-\frac{1}{n}\right)} \frac{\mathcal{F}_{n}(y /(y-1))}{\mathcal{F}_{n}(y)}
$$

with $y$ the four point ratio, $c$ the central charge, and $\mathcal{F}_{n}(x)$ a universal scaling function, which depends on the full operator content of the underlying CFT. The analytical form of $\mathcal{F}_{n}(x)$ is known only for the Luttinger liquid and the $1 \mathrm{D}$ Ising universality class (see Refs. [11, 17, 19] for their precise expression).

$R_{3}(y)$ versus $y$ for hard-core bosons at quarter and half filling is shown in Fig. 5 (a) Different values of $y$ on $x$-axis are obtained by varying the length of the two intervals at fixed $d=L / 2$ (cf. Fig. 1 (c)). The dashed-dotted line is the asymptotic CFT result from Eq. (7). In the limit $y \rightarrow 0$, i.e. two intervals far apart $(d-\ell \rightarrow \infty$ in Fig. 1), one has $\rho_{A_{1} \cup A_{2}} \approx \rho_{A_{1}} \otimes \rho_{A_{2}}$, implying $R_{n} \rightarrow 1$. Oppositely, at $y \rightarrow 1$ the case of two adjacent intervals is recovered, and from Eq. (5) one has $R_{n} \rightarrow 0$.

For finite chains we find oscillating corrections that are similar to those of the mutual information between two disjoint intervals $[14-18,57])$. Under general assumptions, for any $n$ their behavior can be given as

$$
R_{n}(y)=R_{n}^{C F T}(y)+g_{n}^{(q)}(y) \ell^{-\omega_{n}^{\prime}}+\ldots,
$$

with $\omega_{n}^{\prime}$ being the corrections exponent, and $g_{n}^{(q)}(y)$ their amplitude, which depends on both $y$ and the parity $q$ of the interval length (the dots in Eq. (8) denote more irrelevant terms). A standard finite size scaling analysis, fitting QMC data at fixed $y=1 / 2$ to $\sim 1 / \ell^{\omega_{3}^{\prime}}$, gives $\omega_{3}^{\prime}=0.6(1)$, which is consistent

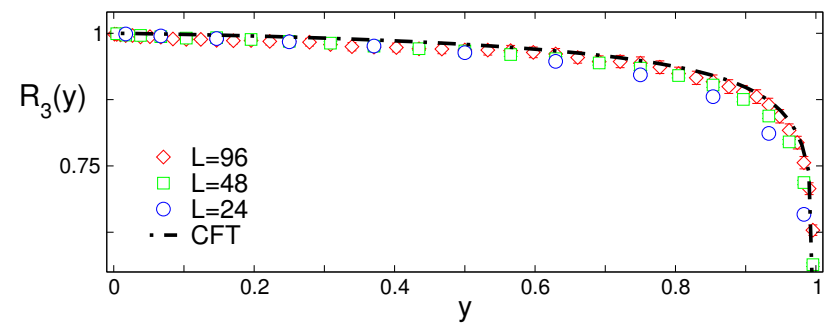

FIG. 6. Bose-Hubbard chain in the superfluid phase: scale invariant ratio $R_{3}(y)$ versus the cross ratio $y$. QMC data are for a periodic chain at $U=2$ (corresponding to Luttinger parameter $K_{L} \approx 3.125$ ), and temperatures $T=0.96 / L$. The dashed-dotted line is the asymptotic zero temperature CFT result.

with $\omega_{3}^{\prime}=\omega_{3}=2 K_{L} / 3=2 / 3$ (see Eq. (6)). This is further supported in Fig. 5 (inset) plotting $d_{3} \equiv R_{3}^{C F T}-R_{3}$ at fixed $y$ versus $\ell^{-2 / 3}$. Dashed lines are one parameter fits to $\sim \ell^{-2 / 3}$. The amplitudes $g_{3}^{(q)}(y)\left(\right.$ extracted as $\left.g_{3}^{(q)} \equiv d_{3} \ell^{2 / 3}\right)$ are shown in Fig. 5 (b), and are rapidly vanishing at $y \rightarrow 0$. Also, data for different sizes collapse on the two curves, confirming a posteriori the correctness of our analysis.

Finally, to demonstrate the versatility of our QMC approach, we discuss $R_{3}(y)$ in the superfluid Bose-Hubbard model on a periodic chain. Already at $L=96$ QMC data are in impressive agreement (at any $0 \leq y \leq 1$ ) with the asymptotic CFT result (dashed-dotted line in the Fig. 6). This confirms that scaling corrections to $R_{n}(y)$ become smaller upon increasing the Luttinger parameter, (similarly to what has been observed in Ref. 30).

Summary \& discussion.- We presented a Quantum Monte Carlo scheme for calculating the moments of the partially transposed reduced density matrix, both at zero and finite temperature. These are the main ingredients in CFT to calculate the logarithmic negativity. We considered several combinations $\left(r_{n}, R_{n}\right)$ of the moments that are scale invariant at a 1D quantum critical point, and, not relying on any local order parameter, could be useful to detect exotic (topological) critical behavior. After taking into account unusual (oscillating) scaling corrections, their behavior is in full agreement with recent CFT results, for both 1D hard-core bosons and the 1D Bose-Hubbard model.

Our results pave the way to many possible research directions. First, it would be interesting to apply the method to higher dimensions, especially to investigate the behavior of $r_{n}, R_{n}$ in topologically ordered phases of matter.

Also, it should be possible to obtain high-temperature series expansions (in any dimension) for the moments of $\rho_{A}^{T_{2}}$ and the negativity itself. Our QMC scheme could then be used as a useful benchmark method.

Acknowledgements. - We acknowledge support by the Austrian Science Fund (FWF) through the SFB FoQuS (FWF Project No. F4018-N23). We acknowledge financial support and allocation of CPU time from NSC and NCTS Taiwan. This work was supported by the Austrian Ministry of Science BMWF as part of the UniInfrastrukturprogramm of the Forschungsplattform Scientific Computing at LFU Innsbruck. 
[1] A. Osterloh, L. Amico, G. Falci, and R. Fazio, Nature 416, 608 (2002).

[2] C. Holzhey, F. Larsen, and F. Wilczek, Nuclear Physics B 424, 443 (1994).

[3] G. Vidal, J. I. Latorre, E. Rico, and A. Kitaev, Phys. Rev. Lett. 90, 227902 (2003).

[4] P. Calabrese and J. Cardy, J. Stat. Mech.: Theor. Exp., P06002 (2004).

[5] L. Amico, R. Fazio, A. Osterloh, and V. Vedral, Rev. Mod. Phys. 80, 517 (2008).

[6] J. Eisert, M. Cramer, and M. B. Plenio, Rev. Mod. Phys. 82, 277 (2010).

[7] P. Calabrese, J. Cardy, and B. Doyon, J. Phys. A: Math. Theor. 42, 500301 (2009).

[8] J. Cardy, J. Stat. Mech.: Theor. Exp. , P10004 (2010).

[9] M. Caraglio and F. Gliozzi, JHEP 11, 076 (2008).

[10] S. Furukawa, V. Pasquier, and J. Shiraishi, Phys. Rev. Lett. 102, 170602 (2009).

[11] P. Calabrese, J. Cardy, and E. Tonni, J. Stat. Mech.: Theor. Exp. 2009, P11001 (2009).

[12] P. Calabrese and J. Cardy, J. Phys. A: Math. Theor. 42, 504005 (2009).

[13] F. Igloi and I. Peschel, EPL 89, 40001 (2010).

[14] M. Fagotti and P. Calabrese, J. Stat. Mech.: Theor. Exp. 2010, P04016 (2010).

[15] M. Fagotti and P. Calabrese, J. Stat. Mech.: Theor. Exp. 2011, P01017 (2011).

[16] M. Fagotti, EPL 97, 17007 (2012).

[17] V. Alba, L. Tagliacozzo, and P. Calabrese, Phys. Rev. B 81, 060411 (2010).

[18] V. Alba, L. Tagliacozzo, and P. Calabrese, J. Stat. Mech.: Theor. Exp. , P06012 (2011).

[19] P. Calabrese, J. Cardy, and E. Tonni, J. Stat. Mech.: Theor. Exp. 2011, P01021 (2011).

[20] A. Coser, L. Tagliacozzo, and E. Tonni, arXiv:1309.2189 (2013).

[21] M. M. Wolf, F. Verstraete, M. B. Hastings, and J. I. Cirac, Phys. Rev. Lett. 100, 070502 (2008).

[22] G. Vidal and R. F. Werner, Phys. Rev. A 65, 032314 (2002).

[23] H. Wichterich, J. Molina-Vilaplana, and S. Bose, Phys. Rev. A 80, 010304 (2009).

[24] H. Wichterich, J. Vidal, and S. Bose, Phys. Rev. A 81, 032311 (2010).

[25] P. Calabrese, J. Cardy, and E. Tonni, Phys. Rev. Lett. 109, 130502 (2012).

[26] P. Calabrese, J. Cardy, and E. Tonni, J. Stat. Mech.: Theor. Exp. , P02008 (2013).

[27] Y. A. Lee and G. Vidal, arxiv:1306.5711 (2013).

[28] C. Castelnovo, Phys. Rev. A 88, 042319 (2013).

[29] F. Gliozzi and L. Tagliacozzo, J. Stat. Mech.: Theor. Exp. , P01002 (2010).

[30] V. Alba, J. Stat. Mech.: Theor. Exp. 2013, P05013 (2013).
[31] M. B. Hastings, I. Gonzalez, A. B. Kallin, and R. G. Melko, Phys. Rev. Lett. 104, 157201 (2010).

[32] R. G. Melko, A. B. Kallin, and M. B. Hastings, Phys. Rev. B 82, 100409 (2010).

[33] R. R. P. Singh, M. B. Hastings, A. B. Kallin, and R. G. Melko, Phys. Rev. Lett. 106, 135701 (2011).

[34] S. V. Isakov, M. B. Hastings, and R. G. Melko, Nature Physics 7, 772 (2011).

[35] R. K. Kaul, R. G. Melko, and A. W. Sandvik, Ann. Rev. Cond. Matt. Phys. 4, 179 (2013).

[36] S. Inglis and R. G. Melko, Phys. Rev. E 87, 013306 (2013).

[37] J. Iaconis, S. Inglis, A. B. Kallin, and R. G. Melko, Phys. Rev. B 87, 195134 (2013).

[38] S. Humeniuk and T. Roscilde, Phys. Rev. B 86, 235116 (2012).

[39] C.-M. Chung, L. Bonnes, P. Chen, and A. M. Läuchli, arXiv:1305.6536 (2013).

[40] F. F. Assaad, T. C. Lang, and F. P. Toldin, arXiv:1311.5851 (2013).

[41] K. Binder, Phys. Rev. Lett. 47, 693 (1981).

[42] K. Audenaert, J. Eisert, M. B. Plenio, and R. F. Werner, Phys. Rev. A 66, 042327 (2002).

[43] J. Cardy and P. Calabrese, J. Stat. Mech.: Theor. Exp. 2010, P04023 (2010).

[44] B. Nienhuis, M. Campostrini, and P. Calabrese, J. Stat. Mech.: Theor. Exp. 2009, P02063 (2009).

[45] P. Calabrese, M. Campostrini, F. Essler, and B. Nienhuis, Phys. Rev. Lett. 104, 095701 (2010).

[46] P. Calabrese and F. H. L. Essler, J. Stat. Mech.: Theor. Exp. 2010, P08029 (2010).

[47] T. D. Kühner and H. Monien, Phys. Rev. B 58, R14741 (1998).

[48] S. Rachel, N. Laflorencie, H. F. Song, and K. Le Hur, Phys. Rev. Lett. 108, 116401 (2012).

[49] A. M. Läuchli, arXiv:1303.0741 (2013).

[50] Choosing $\ell=L / 4$ in the denominator of the definition of $r_{n}(z)$ ensures that non-universal contributions cancel in the thermodynamic limit [25]. This covention leads, for instance, to a crossing of $r_{3}$ at $z=1 / 4$ (see Fig. 3) that must not be taken as an indicator of universality at this specific value of $z$.

[51] N. V. Prokof'ev, B. V. Svistunov, and I. S. Tupitsyn, JETP 87, 310 (1998).

[52] N. V. Prokof'ev, B. V. Svistunov, and I. S. Tupitsyn, Physics Letters A 238, 253 (1998).

[53] L. Pollet, K. V. Houcke, and S. M. A. Rombouts, J. Comp. Phys. 225, 2249 (2007).

[54] P. Calabrese, L. Tagliacozzo, and E. Tonni, J. Stat. Mech.: Theor. Exp., P05002 (2013).

[55] N. Laflorencie, E. S. Sørensen, M.-S. Chang, and I. Affleck, Phys. Rev. Lett. 96, 100603 (2006).

[56] I. Affleck, N. Laflorencie, and E. S. Sørensen, J. Phys. A: Math. Theor. 42, 504009 (2009).

[57] M. Fagotti and P. Calabrese, J. Stat. Mech.: Theor. Exp. 2011, P01017 (2011). 